\title{
Can geostrophic adjustment of baroclinic disturbances in tropical atmosphere explain MJO events?
} \\ ${ }^{a}$ Laboratoire de Météorologie Dynamique (LMD)/IPSL, Sorbonne Université and Ecole Normale Supérieure (ENS), Paris, France \\ ${ }^{b}$ Institute for Geophysics and Meteorology (IGM), University of Cologne, Cologne, Germany
}

This is the Author's Original Manuscript (AOM); that is, the manuscript in its original form; a "preprint". The Version of Record of this manuscript has been accepted for publication by the Quarterly Journal of the Royal Meteorological Society (QJRMS), published by Royal Meteorological Society. The publisher's version is available at https://doi.org/10.1002/qj.3884. 


\title{
Can geostrophic adjustment of baroclinic disturbances in tropical atmosphere explain MJO events?
}

\author{
MASOUD ROSTAMI ${ }^{\dagger} \ddagger$ and VLADIMIR ZEITLIN ${ }^{\dagger} *$ \\ ${ }^{\dagger}$ Laboratoire de Météorologie Dynamique, Sorbonne University (SU)/ Ecole Normale Supérieure (ENS)/CNRS, 75005 Paris, France \\ $\ddagger$ Institute for Geophysics and Meteorology, University of Cologne, 50969 Cologne, Germany
}

\begin{abstract}
Using the two-layer moist-convective rotating shallow water model, we study the process of relaxation (adjustment) of localized large-scale pressure anomalies in the lower equatorial troposphere, and show that it engenders coherent structures strongly resembling the Madden Julian Oscillation (MJO) events, as seen in vorticity, pressure, and moisture fields. We demonstrate that baroclinicity and moist convection substantially change the scenario of the quasi-barotropic "dry" adjustment, which was established in the framework of one-layer shallow water model and consists, in the long-wave sector, in the emission of equatorial Rossby waves, with dipolar meridional structure, to the West, and of equatorial Kelvin waves, to the East. If moist convection is strong enough, a dipolar cyclonic structure, which appears in the process of adjustment as a Rossby-wave response to the perturbation, transforms into a coherent modon-like structure in the lower layer, which couples with a baroclinic Kelvin wave through a zone of enhanced convection and produces, at initial stages of the process, a self-sustained slowly eastward-propagating zonally- dissymmetrical quadrupolar vorticity pattern. At the same time, a weaker quadrupolar structure of opposite sign arises in the upper layer, the whole picture similar to the active phase of the MJO events. The baroclinic Kelvin wave then detaches from the dipole, which keeps slow eastward motion, and circumnavigates the Equator, catching up and interacting with the dipole.
\end{abstract}

Key Words: Geostrophic Adjustment, Madden Julian Oscillation (MJO), Equatorial Modon, Convectively Coupled Equatorial Waves

Received...

\section{Introduction}

Starting from the classical works by Matsuno (1966) and Gill (1980) rotating shallow water (RSW) model on the equatorial beta-plane has become a standard conceptual tool for analyzing large-scale dynamics in the equatorial region. The response of the tropical atmosphere to a localized heating obtained in the framework of this model by Gill (1980) represents a foundational result in tropical meteorology and climatology. One of the advantages of the model is its simplicity, which allows, on the one hand, for an advanced theoretical analysis, and, on the other hand, for robust, low-cost, and computationally friendly numerical schemes. Thus, a thorough theoretical investigation of nonlinear relaxation of large-scale localized pressure anomalies at the equator was performed in the RSW framework in (Le Sommer et al. 2004), and cross-checked with direct simulations using the wellbalanced finite-volume numerical scheme of Bouchut (2007). Such relaxation was called geostrophic adjustment in (Le Sommer et al. 2004), because in the long-wave limit, which corresponds to large

*Corresponding author. Email: zeitlin@Imd.ens.fr, Address: LMD-ENS, 24 Rue Lhomond, 75005 Paris, France 
We start by recalling that $2 \mathrm{RSW}$ is obtained by vertical averaging of hydrostatic primitive equations with pseudo-height as vertical coordinate, and that a self-consistent inclusion of moisture, condensation, and related latent heat release in mc2RSW is achieved by a combination of vertical

zonal to meridional aspect ratios of initial disturbances, the geostrophic balance holds in the leading order, although in general it is not well-defined on the equatorial beta-plane. Notice that the classical results by Gill (1980) were obtained, precisely, in this limit. We should stress that the results of Gill (1980) and Le Sommer et al. (2004) are, in fact, related, as understanding of the initial-value (adjustment) problem allows to anticipate a response to localized forcing. Indeed, both give equatorial Rossby-wave and equatorial Kelvin wave responses to the perturbation/forcing, respectively, to the West and to the East of the perturbation, or forcing.

A further development of the RSW model, by coupling the evolution of humidity and condensation to hydrodynamics, allowed to model the dynamical effects of moist convection and related latent heat release in a simple albeit self-consistent way (Bouchut et al. 2009), while these latter were crudely represented by just a mass sink without any moisture feedback in the pioneering papers (Gill 1980; Heckley and Gill 1984). Recently, such moist-convective RSW (mcRSW) modeling allowed to get new insights into the influences of the moist convection upon dynamical phenomena in tropics, including the Madden-Julian Oscillation (MJO) (Rostami and Zeitlin 2019a,b; Vallis and Penn 2019). We should emphasize that, in spite of the abundant literature, there is no consensus on the dynamical explanation of the MJO, e.g. (Fuchs and Raymond 2017; Majda and Stechmann 2009; Matthews 2000; Sobel and Maloney 2013; Wedi and Smolarkiewicz 2010; Yang and Ingersoll 2011; Yano and Tribbia 2017) and the recent review (Zhang et al. 2020) ${ }^{\dagger}$. However, an essential ingredient, the vertical structure, which is well-pronounced in the MJO events, cf. e.g. Zhang (2005), is missing in the above-cited shallow-water studies, as RSW and mcRSW are, by construction, vertically averaged, and hence quasi-barotropic models. The simplest baroclinic extension of RSW is the well-known two-layer RSW model, 2RSW, cf. e.g. Zeitlin (2018). Its moist-convective extension (mc2RSW) was proposed in (Lambaerts et al. 2011b), and enriched by inclusion of surface evaporation, of liquid water and its entrainment, of vaporization, and of precipitation in (Rostami and Zeitlin 2018). Relaxation of localized initial perturbations, the equatorial adjustment process, is much less studied in the baroclinic models (although such studies exist, e.g. (Hoskins and Jin 1991)) than in one-layer shallow water. For example, we are not aware of a baroclinic analog of nonlinear equatorial adjustment theory of Le Sommer et al. (2004). However, we can anticipate that, at least in the long-wave limit, the scenario of adjustment in 2RSW would consist in generation of faster barotropic and slower baroclinic equatorial Rossby and Kelvin waves propagating, respectively, westwards and eastwards from the initial perturbation. Yet, for intense initial pressure perturbations with zonal to meridional aspect ratio close to one, and in the presence of condensation, such standard scenario was shown to be replaced the one-layer mcRSW by another one, producing eastward-propagating cyclonic dipoles, the equatorial modons in the Rossby-wave sector (Rostami and Zeitlin 2019b). The question, thus, arises how the latter scenario is modified in the presence of baroclinicity.

The purpose of the present paper is to study nonlinear equatorial adjustment of localized, largescale, baroclinic pressure perturbations in moist-convective environment using the simplest version of mc2RSW, and to explore its possible link with MJO. As we will show, the results are rather surprising, and exhibit, in certain ranges of parameters, dynamical structures strikingly resembling the MJO events.

The paper is organized as follows: in Section 2 we present the mc2RSW model, sketch its basic properties, and analyze its linear-wave spectrum. In Section 3 we present and analyze the results of numerical simulations with finite-volume scheme, which reveal the MJO-like structures. Section 4 contains a summary of the results and a discussion of the tentative link to the MJO events.

\section{The mc2RSW model and its linear wave spectrum}

${ }^{\dagger}$ which appeared after the present paper was submitted 
averaging between pairs of isobaric surfaces and Lagrangian conservation of the linearized equivalent potential temperature (Lambaerts et al. 2011b). The equations of the model with a moist lower and dry upper layer read:

$$
\begin{aligned}
& \partial_{t} \boldsymbol{v}_{1}+\left(\boldsymbol{v}_{1} \cdot \boldsymbol{\nabla}\right) \boldsymbol{v}_{1}+f(y) \hat{\boldsymbol{z}} \times \boldsymbol{v}_{1}=-g \boldsymbol{\nabla}\left(h_{1}+h_{2}\right), \\
& \partial_{t} \boldsymbol{v}_{2}+\left(\boldsymbol{v}_{2} \cdot \boldsymbol{\nabla}\right) \boldsymbol{v}_{2}+f(y) \hat{\boldsymbol{z}} \times \boldsymbol{v}_{2}=-g \boldsymbol{\nabla}\left(h_{1}+s h_{2}\right)+\frac{\boldsymbol{v}_{1}-\boldsymbol{v}_{2}}{h_{2}} \gamma C, \\
& \partial_{t} h_{1}+\boldsymbol{\nabla} \cdot\left(h_{1} \boldsymbol{v}_{1}\right)=\underline{-\gamma C}, \\
& \partial_{t} h_{2}+\boldsymbol{\nabla} \cdot\left(h_{2} \boldsymbol{v}_{2}\right)=\underline{+\gamma C}, \\
& \partial_{t} Q+\boldsymbol{\nabla} \cdot\left(Q \boldsymbol{v}_{1}\right)=\underline{-C+E},
\end{aligned}
$$

Here $\boldsymbol{v}_{i}=\left(u_{i}, v_{i}\right)$ is the horizontal velocity field in layer $i=1,2$ (counted from the bottom), $f(y)$ is the Coriolis parameter, which is equal to $\beta y$ on the equatorial beta-plane, $\hat{z}$ is the unit vector in vertical direction, $h_{i}$ are the thicknesses of the layers, with the values at rest $H_{i}$, correspondingly, $\theta_{i}$ is the normalized potential temperature in each layer, $g$ is the gravity acceleration, and $s=\theta_{2} / \theta_{1}>1$ is the stratification parameter. Without the underlined terms (2.1) is the standard non-dissipative twolayer atmospheric shallow-water model, cf. Zeitlin (2018), with an addition of a passive scalar, the bulk water vapor content in the lower-layer column $Q$, which is conserved if diabatic effects are switched off. We will call the system "dry" in this case. The underlined terms introduce a condensation sink $C$ and a surface evaporation source $E$ in the moisture conservation equation (2.1e), and a convective flux due to the latent heat release, which is proportional to the condensation, between the layers in mass conservation equations (2.1c), (2.1d). The proportionality coefficient $\gamma$ is defined by the underlying vertical stratification and thermodynamical parameters, cf. (Lambaerts et al. 2011b). The convective flux leads to a Rayleigh drag appearing in (2.1b). As discussed in (Rostami and Zeitlin 2018), the drag repartition between the layers is not unique. We choose here the original version of Lambaerts et al. (2011b), which gives in the limit $h_{2}>>h_{1}$ the one-layer mcRSW we referred to in the Introduction. We use the relaxational parametrization for condensation, with a relaxation time $\tau$ :

$$
C=\frac{Q-Q^{s}}{\tau} \mathcal{H}\left(Q-Q^{s}\right),
$$

where $\mathcal{H}$ denotes the Heaviside (step-) function, and the bulk formula for surface evaporation:

$$
E=\alpha \frac{\left|\boldsymbol{v}_{1}\right|}{\left|\boldsymbol{v}_{\max }\right|}\left(Q^{s}-Q\right) \mathcal{H}\left(Q^{s}-Q\right),
$$

where $\left|\boldsymbol{v}_{\max }\right|$ is the maximum value of the lower-layer velocity, and $\alpha$ is an adjustable coefficient. Parametrization of the condensation (2.2) is of the Betts-Miller type (Betts and Miller 1986), used in general circulation models, and the parametrization of evaporation (2.3) is standard in modeling ocean-atmosphere exchanges (Katsaros 2001), except for renormalization by the maximum velocity, which is convenient. In the simplified version, the saturation value $Q^{s}$ can be taken to be constant, although it depends on pressure, i.e. on $h$ in the present context, according to Clausius-Clapeyron law, cf. (Bouchut et al. 2009). Evaporation (2.3) is the only influence of the boundary layer we include in the model. Momentum dissipation due to bottom friction can be easily included, and more involved parameterizations of the boundary layer can be used, cf. (Schecter and Dunkerton 2009), which we will not do, preferring to keep the model as simple as possible. We should also emphasize that radiative relaxation can be easily incorporated in the model as well, which is not done here, by the same reason.

Let us emphasize that, compared to the so-called WISHE models (see a shallow-water version of such model, and key references in (Solodoch et al. 2011)), a class of simplified models representing dynamical role of moisture and convection which are applied also to MJO, e.g. (Fuchs and Raymond 2017), the mcRSW models fully retain the hydrodynamic nonlinearity, which is important, as will be clear from the following. As to evaporation, which is a key element of WISHE models, it is not 
indispensable in mcRSW, as even without it the model captures well, for example, all basic stages of the development of the moist baroclinic instability (Lambaerts et al. 2012).

As is easy to check, in the absence of evaporation, which acts as a forcing, the model conserves total mass and momentum, as well as the moist enthalpy of the lower, moist, layer $M=h_{1}-\gamma Q$. M should be positive- definite, see below. General properties of the model, and analysis of the conservation laws can be found in (Rostami and Zeitlin 2017). It is worth recalling an important for the interpretation of the numerical simulations fact that convective flux enhances cyclonic vorticity in the moist-convective layer, see the demonstration in (Lambaerts et al. 2011a).

As was already said in the Introduction, baroclinic and barotropic equatorial waves are expected as an output of the adjustment process, which will be studied below in the mc2RSW model. In the next subsection we derive the spectrum of linear waves in the model in the long-wave approximation.

\subsection{Linear long-wave spectrum}

As is well-known, cf. e.g. (Zeitlin 2018), in the "dry" 2RSW the spectrum of linear waves of the onelayer RSW duplicates, each wave species having corresponding barotropic and baroclinic modes. In the "moist" system (2.1), the condensation $C$ is a nonlinear function of $Q$. Yet, in the limit of immediate relaxation, which is pertinent in the tropics as the relaxation time is, typically, few hours for deep convection, which is short compared to characteristic evolution times of large-scale systems like MJO, equation (2.1e) in the absence of evaporation becomes

$$
\tau \rightarrow 0 \Rightarrow C \rightarrow-Q_{s} \boldsymbol{\nabla} \cdot \boldsymbol{v},
$$

and the whole system allows then for a straightforward linearization. The idea of immediate relaxation, and of the simplification (2.4) it produces, goes back to Gill (1982). The mathematical properties of the resulting system were thoroughly analyzed in (Lambaerts et al. 2011b). To get the wave spectrum, we use the long-wave scaling of (Le Sommer et al. 2004), which we adapt to the two-layer system. Notice that this scaling is not the one adopted in numerical simulations below. We introduce a meridional scale $L$ and the meridional to zonal aspect ratio $\delta$, which we suppose to be small. We also introduce a slow time-scale T:

$$
y \sim L, \quad x \sim \delta^{-1} L, \quad t \sim T=(\delta \beta L)^{-1}, \quad \delta \ll 1 .
$$

The velocity is scaled as

$$
u_{i} \sim U, \quad v_{i} \sim \delta U
$$

and we introduce nonlinearity parameters governing the deviations $\eta_{i}$ of $h_{i}$ from their rest values:

$$
h_{i}=H_{i}\left(1+\lambda_{i} \eta_{i}\right) \text {. (no summation over i) }
$$

As in (Le Sommer et al. 2004), we will consider a parameter regime where nonlinearity and Rossby number are of the same order:

$$
\lambda_{1} \sim \lambda_{2} \sim \epsilon=\frac{U}{\beta L^{2}}, \frac{g H_{1}}{\left(\beta L^{2}\right)^{2}} \sim 1 .
$$

Here $\epsilon$ is the equatorial Rossby number. With this scaling, and under hypothesis of immediate relaxation (2.4), the equations (2.1) take the form

$$
\left\{\begin{array}{l}
\partial_{t} u_{i}+\epsilon\left(\boldsymbol{v}_{i} \cdot \boldsymbol{\nabla}\right) u_{i}-y v_{i}=-\partial_{x}\left(\eta_{1}+s^{i-1} d \eta_{2}\right)+\mathcal{O}\left(\epsilon^{2}\right) \\
\delta^{2}\left[\partial_{t} v_{i}+\epsilon\left(\boldsymbol{v}_{i} \cdot \boldsymbol{\nabla}\right) v_{i}\right]+y u_{i}=-\partial_{y}\left(\eta_{1}+s^{i-1} d \eta_{2}\right)+\mathcal{O}\left(\delta \epsilon, \epsilon^{2}\right) \\
\partial_{t} \eta_{i}+\left[1+(-1)^{i} \frac{\gamma Q^{s}}{H_{i}}\right] \boldsymbol{\nabla} \cdot \boldsymbol{v}_{i}+\epsilon \boldsymbol{\nabla} \cdot\left(\eta_{i} \boldsymbol{v}_{i}\right)=0+\mathcal{O}\left(\delta \epsilon, \epsilon^{2}\right),
\end{array}\right.
$$

where $i=1,2$. Note the appearance in these equations of the combinations $\gamma Q^{s} / H_{i}$ which characterize the convective activity. We will use the parameter $\kappa=\gamma Q^{s} / H_{1}$ in what follows. We introduced here the notation $d=H_{2} / H_{1}$ for the ratio of non-perturbed thicknesses, and wrote down separately the 
equations for the zonal and meridional components of velocity and thickness perturbations, omitting the higher-order terms. Following (Le Sommer et al. 2004), we assume that

$$
\epsilon \sim \delta^{2}
$$

In the leading order in $\epsilon$ we thus get a linear system:

$$
\left\{\begin{array}{l}
\partial_{t} u_{i}-y v_{i}=-\partial_{x}\left(\eta_{1}+s^{i-1} d \eta_{2}\right) \\
+y u_{i}=-\partial_{y}\left(\eta_{1}+s^{i-1} d \eta_{2}\right) \\
\partial_{t} \eta_{i}+\left[1+(-1)^{i} \frac{\gamma Q^{s}}{H_{i}}\right] \nabla \cdot \boldsymbol{v}_{i}=0
\end{array}\right.
$$

A linear transformation of the form:

$$
\boldsymbol{v}_{ \pm}=\boldsymbol{v}_{1}+A_{ \pm} \boldsymbol{v}_{2}, \eta_{ \pm}=\eta_{1}+B_{ \pm} \eta_{2},
$$

can be used to diagonalize the system (2.10):

$$
\left\{\begin{array}{c}
\partial_{t} u_{ \pm}-y v_{ \pm}+a_{ \pm} \partial_{x} \eta_{ \pm}=0 \\
\partial_{t} v_{ \pm}+y u_{ \pm}+a_{ \pm} \partial_{y} \eta_{ \pm}=0 \\
\partial_{t} \eta_{ \pm}+b_{ \pm} \boldsymbol{\nabla} \cdot \boldsymbol{v}_{ \pm}=0
\end{array}\right.
$$

where

$$
a_{ \pm}=1+A_{ \pm}, \quad b_{ \pm}=1-\kappa .
$$

The details of this transformation can be found in the Appendix. We should only mention that for statically stable configurations with $s>1$ the product $a_{ \pm} b_{ \pm}$is positive, and the system is hyperbolic, if the linearized moist enthalpy $M=H_{1}-\gamma Q^{s}$ is positive. The subscript $-(+)$ corresponds to the baroclinic (barotropic) mode. As was shown in (Lambaerts et al. 2011b) the moist baroclinic modes are always slower than the "dry" ones. $A_{ \pm}$and $B_{ \pm}$are close to -1 for weak stratifications, when $s$ is close to one, and thickness ratios of the order 1.

Following the demonstration presented in (Le Sommer et al. 2004) (see also (Zeitlin 2018), ch. 5 ), it can be straightforwardly shown that general solution of the system (2.12), for both barotropic and baroclinic modes, consists of a mean zonal flow, which we will not consider in the following, equatorial Kelvin waves with a meridional structure $\phi_{0}\left(y / \sqrt{c_{ \pm}}\right)$propagating eastward with the phase velocity

$$
c_{ \pm}=\sqrt{a_{ \pm} b_{ \pm}}
$$

and equatorial Rossby waves with meridional structure $\phi_{n}\left(y / \sqrt{c_{ \pm}}\right)$propagating westward with the phase velocities

$$
c_{n}=\frac{c_{ \pm}}{2 n+1}, \quad n=1,2, \ldots .
$$

Here $\phi_{n}$ are Gauss-Hermite (parabolic cylinder) functions which describe, as is well-known, the meridional structure of waves on the equatorial beta-plane.

To summarize, the spectrum of long linear waves in the model consists of fast barotropic, and slow baroclinic Kelvin and Rossby modes, the former propagating eastward with phase velocities at least three times larger than the latter, which propagate westward.

\section{Numerical simulations of the baroclinic equatorial adjustment}

\subsection{Initialization and the choice of parameters}

In the numerical experiments we we model the relaxation of a localized depression in the lower troposphere over the warm pool, a zone of enhanced uniform humidity. The initial humidity $Q_{i}$ in the lower layer of the mc2RSW model was, thus, chosen to be uniform, and close to the saturation limit $Q^{s}$. The non-dimensional values in the main experiment presented below were taken as $Q^{s}=0.88$, 
$Q_{i}=Q^{s}-0.01$ but other values of $Q^{s}$ were also considered. Initial pressure distributions in the layers $h_{i}=H_{i}(x, y)$ were obtained from the so-called alpha-Gaussian profile, e.g. (Rostami and Zeitlin 2017) centered at the Equator. The convenience of this profile is that it allows to regulate both the size and the steepness of the distribution. The details of the adjustment process could be sensitive to the latter (Rostami and Zeitlin 2019b), although studying this sensitivity is out of the scope of the paper. After normalization by the mean thickness of the layer, an axisymmetric alpha-Gaussian thickness distribution is given by the formula

$$
H_{i}(r, \sigma)=1+\lambda_{i} \frac{2^{1 / \sigma}}{s} \Gamma\left(\frac{1}{\sigma}+\frac{1}{2}\right) G\left(\frac{r^{\sigma}}{2}, \frac{1}{\sigma}+\frac{1}{2}\right),
$$

where $r=\sqrt{x^{2}+y^{2}}, G(r, b)=[1 / \Gamma(b)] \int_{r}^{b} e^{-t} t^{b-1} d t$, and $\Gamma$ denotes the gamma-function. The parameters $\lambda_{i}$ and $\sigma$ determine the amplitude and the steepness of the pressure anomaly, the maximum value of the anomaly $\Delta H / H s$, where $H_{s}$ is a non-perturbed total depth, depending on both. In general, $\lambda_{i}$ can be positive or negative, but below we concentrate on negative pressure perturbations, and only in the lower layer. By stretching the zonal coordinate $x \rightarrow a^{-1} x$ in $H_{i}(x, y)$ we get a perturbation with the zonal to meridional aspect ratio $a$. The amplitude, the steepness, and the aspect ratio of the initial perturbation are variable parameters. The steepness in this study is fixed to be $\sigma=3$. The zonal extent of the anomaly in the simulations we present below is taken to be $\approx 4 L_{d}$, where $L_{d}$ is the barotropic equatorial deformation radius $L_{d}^{2}=\sqrt{g H_{s}} / \beta$, and $H_{s}=\left(H_{1}+H_{2}\right)$. The non-dimensional values of $H_{i}$ were taken as $H_{1}=0.35$ and $H_{2}=0.65$. The simulations were performed with the finite- volume method of Bouchut and Zeitlin (2010) on a regular $500 \times 500$ grid in the domain $11 L_{d} \times 12 L_{d}$ on the equatorial beta-plane. The zonal extent of the domain corresponds to the length of the Equator, and we use periodic boundary conditions in this direction. The meridional extent is exaggerated, in order to be able to implement efficiently the sponge boundary conditions, absorbing short and fast inertiagravity waves produced during the adjustment. The non-dimensional values of parameters, with spatial coordinates measured in units of $L_{d}$, and velocities measured in units of $\sqrt{g H_{s}}$, were chosen as follows: $\beta=1, \gamma=0.35, \alpha=0.15, \tau=90 \Delta t$, where $\Delta t$ is the time-step of the numerical scheme, taken to be small to satisfy the CFL condition: $\Delta t=10^{-3} T$, where $T=L_{d} / \sqrt{g H_{s}}$ is the fast time-scale of the problem. Notice that, according to the definition of $\gamma$ in (Lambaerts et al. 2011b), its non-dimensional value is determined by the non-dimensional thickness of the lower layer. As for the value of the evaporation coefficient $\alpha$, our choice is consistent with the empirical value $\sim 10^{-3}$ of the dimensional transmission coefficient in the bulk evaporation formula (Katsaros 2001), taking into account vertical integration and renormalization by $\left|\mathbf{v}_{\max }\right|$. We choose a slab of thickness $H_{s}=8500 \mathrm{~m}$, to represent the lower part of the tropical troposphere aloft of the boundary layer. The lower, moist, layer of this slab then spreads up to $\approx 5 \mathrm{~km}$ which, roughly, corresponds to the observed vertical extent of the moist layer in MJO events, cf. e.g. (Kiladis et al. 2005). With this choice, the dimensional values of the parameters are $L_{d} \approx 3540 \mathrm{~km}, T \approx 3 \mathrm{~h} 25 \mathrm{~m}$.

\subsection{Results on adjustment of a lower-layer depression}

The goal of the present paper is not to present an exhaustive analysis of the equatorial adjustment in the two-layer system, which will be given elsewhere but, rather, to pinpoint the existence of parameter regimes where this process produces the MJO-like events. The results of the adjustment of one of such cases, for an initial depression in the lower layer with aspect ratio $a=2$ and intensity $\Delta H / H_{s}=0.07$, are presented in Fig. 1. The initial depression is chosen to be relatively substantial, a roughly $600 \mathrm{~m}$ dip in the interface, meaning that the adjustment is, indeed, nonlinear. As in the one-layer case, cf, (Rostami and Zeitlin 2019b), there is a threshold $\Delta H / H_{s} \approx 0.06$, below which only a standard scenario of Rossby-wave emission in the western sector is realized, although in the one-layer case this threshold is much higher. As follows from the Figure, at the onset of the adjustment process a quasi-isotropic inflow in both layers starts towards the center of the initial depression. The winds then deviate from the center because of the action of the Coriolis force, creating cyclonic vorticity zones at both sides of the 

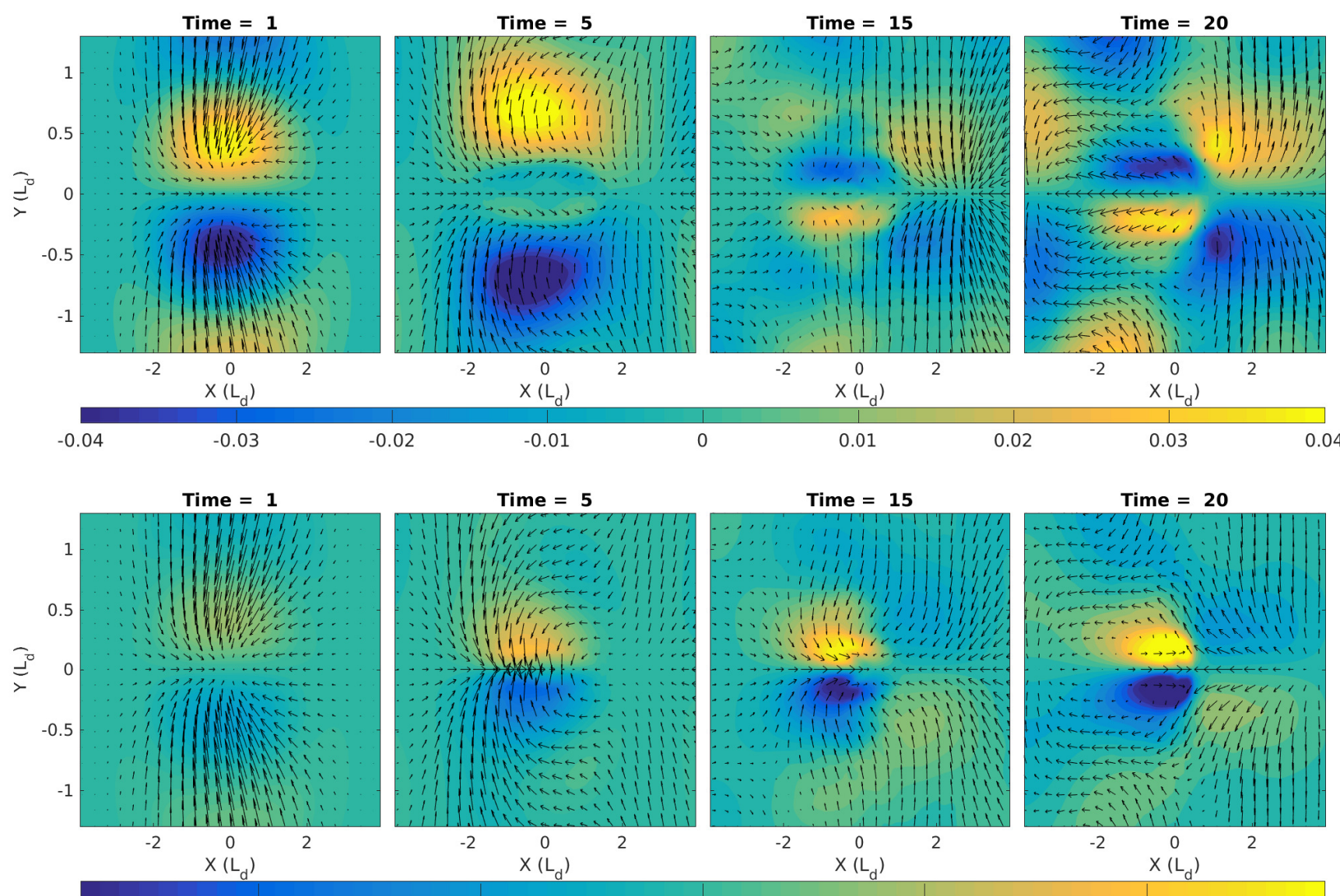

$-0.1$

$-0.05$

0

0.05

0.1
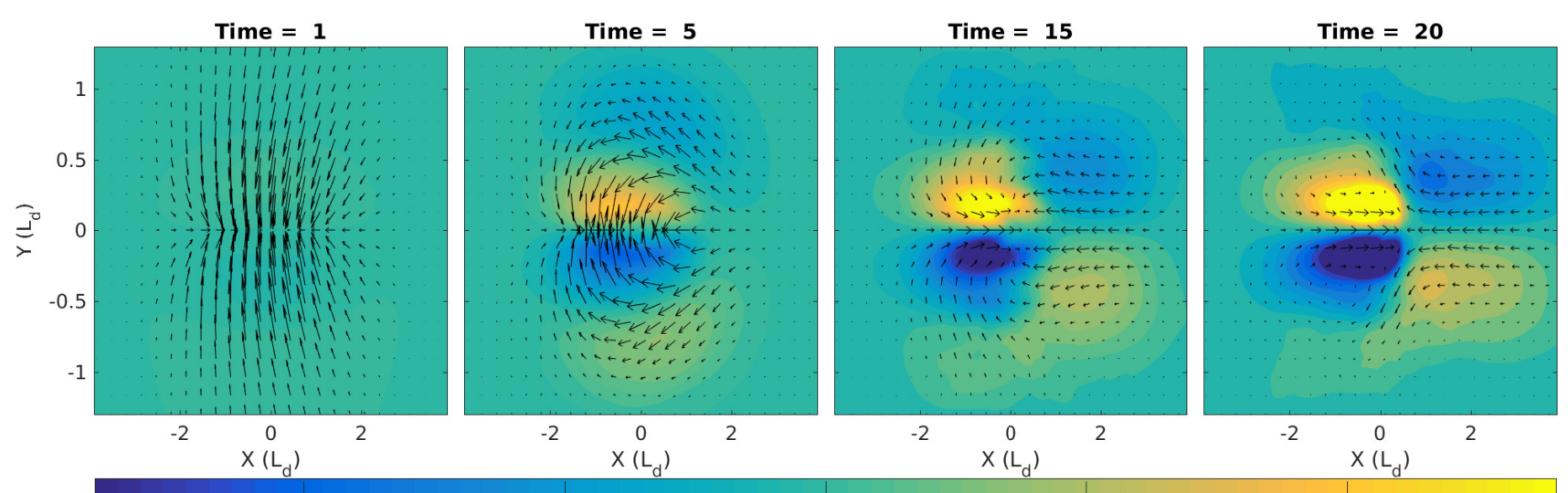

$-0.1$

$-0.05$

0

0.05

0.1

Figure 1. Equatorial adjustment of a negative pressure anomaly in the moist lower layer, and unperturbed dry upper layer, as seen in the initial evolution of velocity (arrows) and relative vorticity (colors) fields. The horizontal panels correspond to the upper-layer, lower-layer, and baroclinic components, respectively from top to bottom. Horizontal scale: equatorial deformation radius $\left(L_{d} \approx 3540 \mathrm{~km} \approx 32^{\circ}\right.$ longitude $)$. Total height $H_{s}=8500 \mathrm{~m}$. Time-scale: $\left[\left(L_{d} / \sqrt{g H_{s}}\right) \approx 0.14 d a y\right]$. $H_{1}=0.35, H_{2}=0.65, \Delta H=0.07 H, \gamma($ latent heat release coefficient $)=0.35, Q^{s}=0.88$. Initial $Q=Q^{s}-0.01$.

the Equator. These vorticity anomalies, due to the moist convection, are then intensified, especially the lower-layer one, and, therefore, the baroclinic vorticity component (notice the difference in the color codes in the upper, middle, and lower panels of the Figure). Later on, a weaker vorticity dipole of the opposite sign, which is attached to the main dipole at its eastern edge, builds up forming a zonally dissymmetrical quadrupole. At the same time a weaker quadrupole of the opposite sign arises in the upper layer, and the whole system starts slowly moving eastwards, as will be discussed more below.

This process can be compared to the adjustment of similar initial anomalies in the one-layer mcRSW, which was recently described in (Rostami and Zeitlin 2019b), and also with earlier results of (Le Sommer et al. 2004). As was shown in these papers, in the case of "dry" adjustment, a localized pressure anomaly, after emitting a packet of fast and short inertia-gravity waves, produces an eastwardpropagating Kelvin, and a westward-propagating Rossby wave packets in the long wave regime, i.e. for 
zonally elongated initial anomalies. If the initial perturbation is not elongated, the signal of adjustment in the western sector can be dominated by westward-propagating large-scale inertia-gravity wave packet (Rostami and Zeitlin 2019b). For strong enough initial perturbations, with much higher than in the present case $\Delta H / H$, the moist-convective adjustment can lead to formation of eastward-moving convection-coupled compact cyclonic dipoles, the equatorial modons (Rostami and Zeitlin 2019a).

In the two-layer framework in the "dry" case, for an essentially baroclinic, that is strongly different layer-wise which is our case here, initial perturbations of small to moderate amplitude, we can expect, on the basis of these results, and of the linear analysis of section 2.1, a response with a strong signature of baroclinic eastward-propagating Kelvin and westward-propagating Rossby waves, with an admixture of westward-propagating long inertia-gravity waves in the case of order one aspect ratios.

In order to check this expectation, we performed a simulation of the "dry" adjustment with the same initial conditions. A comparison of adjustments in the "dry" and moist-convective environments, as seen in the baroclinic velocity and pressure fields, defined in (2.11), is presented in Fig. 2. As follows from the Figure, the "dry" adjustment, indeed, produces Kelvin and Rossby wave packets, which are split at $t \approx 20$, and then continue to further separate, Kelvin wave moving eastward, and Rossby wave moving westward (not shown). Notice also a depression westward from the Rossby-wave dipole, with a characteristic for westward-propagating equatorial inertia-gravity wave (WIGW) velocity pattern. In contrast with the "dry" case, where they split, in the moist-convective environment the baroclinic Kelvin and Rossby waves fusion and form a hybrid mode, which starts a slow eastward motion as a whole. The moist convection is clearly at the origin of this fusion, as it is enhanced at the junction between the Rossby-wave dipole and the Kelvin wave, compare the panels at $t=15$ of Figs. 1, 2 and of Fig. 3, where we display the related evolution of condensation and divergence fields. The resulting hybrid mode is in striking resemblance with the response to a localized heating predicted in (Gill 1980), with an essential difference that here we are dealing with a non-forced (except for evaporation) initial value problem, and the resulting coherent structure is self-sustained and self-propelling eastwards, while Gill (1980) considered a stationary forced problem.

In what concerns the Rossby-wave part of the response, its transition to eastward-propagating dipolar structure under the influence of moist convection is similar to that observed in the barotropic case, cf. Rostami and Zeitlin (2019b). However, here it happens for much weaker (and, hence, more realistic) amplitudes of the perturbation. Yet, its fusion with the Kelvin wave is a purely baroclinic effect. Indeed, the barotropic Kelvin wave is fast and rapidly splits off the Rossby-wave dipole in the one-layer model, while the baroclinic Kelvin wave is slow, and it has time to couple to the Rossby-wave dipole through convection. Enhanced convergence at the rear of the Kelvin wave, cf. the lower panel at $t=15$ of Fig. 3 , is bringing moisture and enhances the effect of the convergence proper to the dipole. Notice also an enhanced condensation related to the WIGW westward of the main dipole at the early stages of adjustment. The weaker secondary dipole, which forms with the main dipole the quadrupole in the lower panel of Fig. 1 corresponds to the relative vorticity distribution in the Kelvin wave. The weaker quadrupole of opposite sign in the upper layer, which forms in the process of "dry" adjustment, as well (not shown), is enhanced due to the opposite sign of the condensation term in the corresponding mass conservation equation in (2.1). The whole thus resulting structure is of planetary scale, self-sustained, and slowly self-propelling eastward. The lower-level westerly inflow to the West of the structure and an easterly inflow to the East, both proper to the respective Rossby dipole and depression Kelvin wave, feed the zone of high convergence in the middle, cf Fig. 3, lower panel; its counterpart in the upper layer produces a divergent outflow.

The above-described coherent structure, although long-living, nevertheless evolves. Its Kelvin part starts detaching at $t \approx 25$, leaving behind a dipole which, in turn, keeps its coherence for a long time, cf. Fig. 4. The phase speed of the front edge of the detaching Kelvin wave differs from the speed of the dipole, as follows from the Fig. 5, where we present the Hovmöller diagrams of the lower-layer, upper-layer, baroclinic, and barotropic zonal velocities at the Equator, at $y=0$. The phase velocity of the baroclinic Kelvin wave, which is identified in the corresponding diagram, is in good agreement with the value of $c_{-} \approx 0.081$ in non-dimensional terms, obtained for the values of parameters $d$ and $\kappa$ 

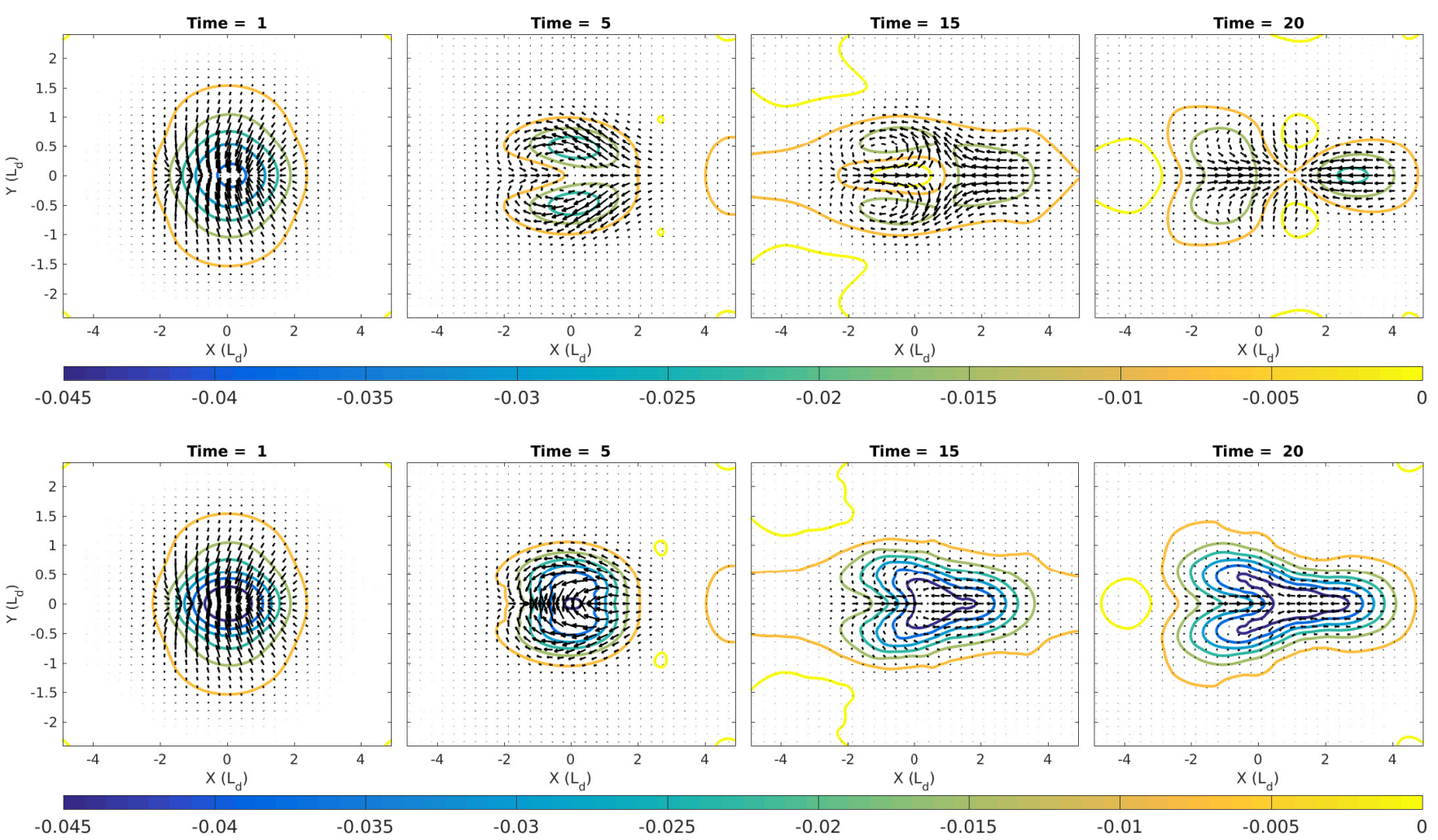

Figure 2. Initial stages of equatorial adjustment of a negative pressure anomaly in the lower layer as seen in evolution of the baroclinic velocity (arrows) and pressure anomaly (contours) in "dry" (upper panel) and moist-convective (lower panel) environments. Same initial configuration as in Fig. 1.

used in the simulation, $\mathrm{cf}$ (2.14) and (A.19), after corresponding renormalizations. This phase velocity is much lower than that of the barotropic Kelvin wave, of which the passages over the domain are seen in upper-row panels, and bottom-right panel of Fig. 5 as small-slope stripes (smaller-scale stripes correspond to barotropic inertia-gravity waves produced by the adjustment). As follows from Fig. 4 the dipole keeps its eastward propagation, although with smaller than at the initial stages of adjustment speed, and eventually stops at $t \approx 85$, cf. the lower-left panel of Fig. 5. The Figure also confirms the opposite sign, and lower intensity, of the dipole in the upper layer at the initial stages, with the opposite sign of its axial velocity. The dipolar coherent structure, clearly identified in the upper row of Fig. 4, and the condensation pattern in the second row, strongly resemble the moist-convective barotropic equatorial modon described in (Rostami and Zeitlin 2019a). Moreover, its intensity and size increase with time, as well as its barotropic component, as is clear from the comparison of upper-left and upper-right panels of Fig. 4. These results seem, at the first glance, paradoxical, as we have recently shown, both theoretically and numerically, that essentially baroclinic modon solutions do not exist in the 2RSW model (Rostami and Zeitlin 2020), only the quasi-barotropic ones can be realized. However, the would-be modon we observe here, first, eventually ceases its eastward propagation, cf Fig. 5, so it is, in fact, a long-living transient. Second, it has a tendency for barotropization, cf. lower-right panel of Fig. 5, which could explain its longevity, according to the results of (Rostami and Zeitlin 2020), and last, but not least, it is not free, but interacting with inertia-gravity waves, and barotropic and baroclinic Kelvin waves, which are clearly visible in Fig. 5. The interaction with the latter is distinctly seen in the upper and lower panels of Fig. 4. The pressure, velocity and divergence patterns allow to easily identify the baroclinic Kelvin wave, with its front part emerging from the West, after having made a tour of the Equator, in the left panels, and its rear, with a characteristic convergence zone already passed over the dipole in the right panels. As is clear from the Figure, the detachment of the Kelvin wave is never complete, with a significant easterly flow remaining in front of the dipole all the time. Hence, the results of (Rostami and Zeitlin 2020) on "free" modons cannot be directly extrapolated to the observed configuration. Note also a slowly expanding westward Rossby wave-like tail behind the dipole, and an extended related drying zone (cf. the third row of the Figure). 


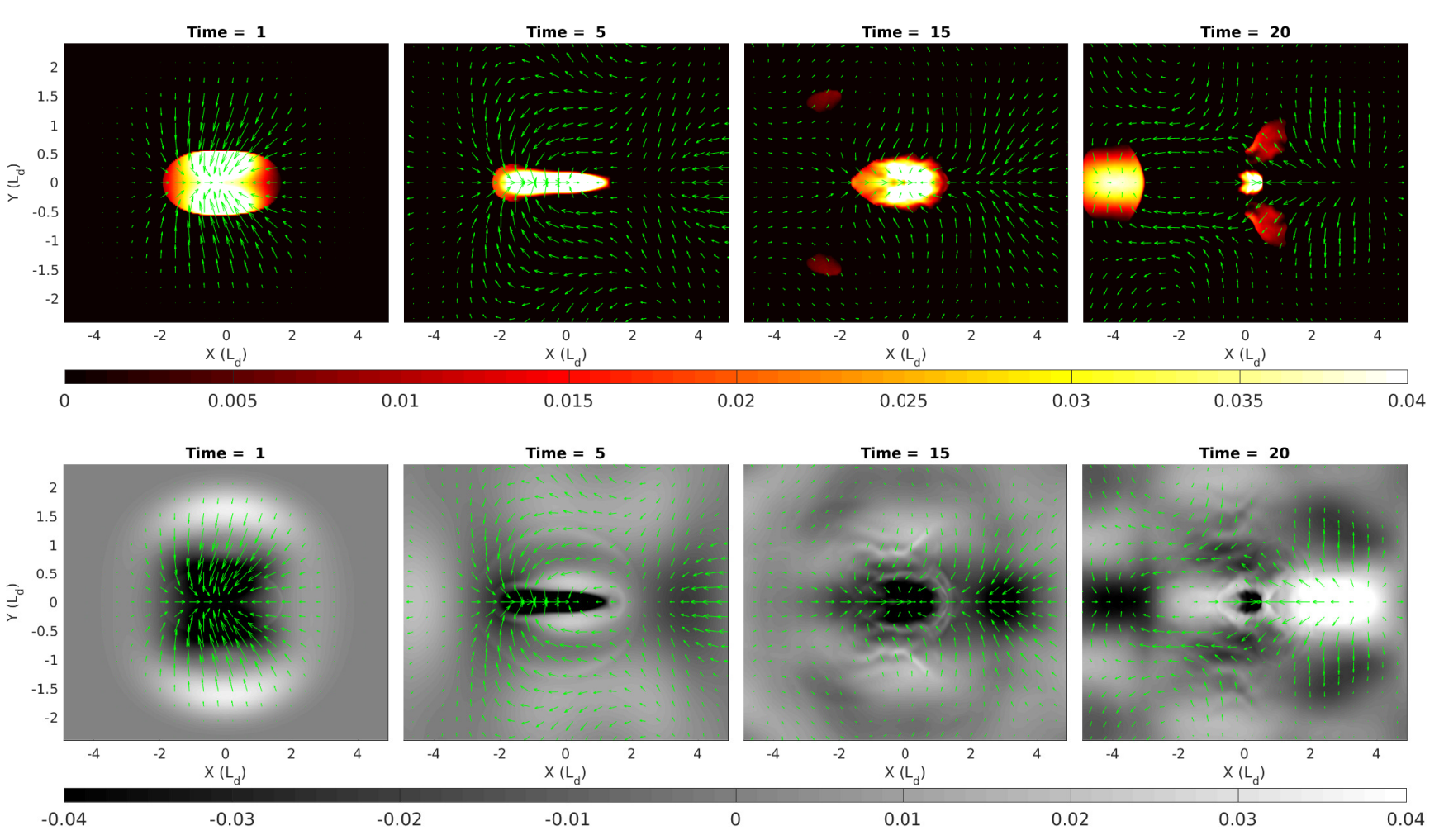

Figure 3. Initial stages of equatorial adjustment of a negative pressure anomaly in the lower layer as seen in the evolution of the lower layer velocity (arrows) and condensation (colors) (upper row) and divergence (lower row). Same initial configuration as in Fig. 1.

Although we presented above the results of only one simulation, we performed a number of them, in particular studying the dependence of the results on stratification and convective activity. The results for the relative vorticity integrated over the domain in simulations with different values of parameters $s$ and $\kappa$ are presented in Fig. 6, with median curves corresponding to the simulation presented in previous figures. As follows from the Figure, decreasing intensity of convective fluxes and/or stratification leads to depletion of vorticity generated by the adjustment process, which can become insufficient to maintain the eastward-moving Rossby dipole (not shown). This, once more, shows that the process of adjustment described above is, essentially, baroclinic.

\section{Summary, discussion, and conclusions}

\subsection{Summary of the results}

Our numerical simulations of the equatorial adjustment of zonally elongated, with aspect ratio 2 lower-level depressions in the two-layer moist-convective rotating shallow water model showed that the behavior of the equatorial Rossby and Kelvin waves, which constitute the main response of the equatorial atmosphere to the perturbation in the long-wave sector, is substantially modified by the effects of moist convection. While these two wave species separate and move, respectively, westward and eastward from the initial perturbation in the adiabatic ("dry") environment, as expected from the previous studies of the equatorial adjustment in the one-layer model, the moist convection, once convective activity is strong enough, allows for fusion of the baroclinic Rossby- and Kelvin- wave parts of the initial response, and formation of a hybrid mode slowly moving eastward and keeping its coherence for a long time which is the most important result of this paper. fusion of Rossby and Kelvin waves becomes possible because the condensation reduces the phase-speed of already slow baroclinic Kelvin wave, and enhances the Rossby-wave dipole to the point that it can propagate eastward, like an equatorial modon. At the same, time, the maximum of convergence and resulting moisture accumulation are situated at the rear of the depression Kelvin wave, which facilitates coupling to the Rossby dipole, with the maximum of convergence and condensation at its front. Still, the size of convective regions is much smaller than the overall circulation pattern. As a result of the fusion, 

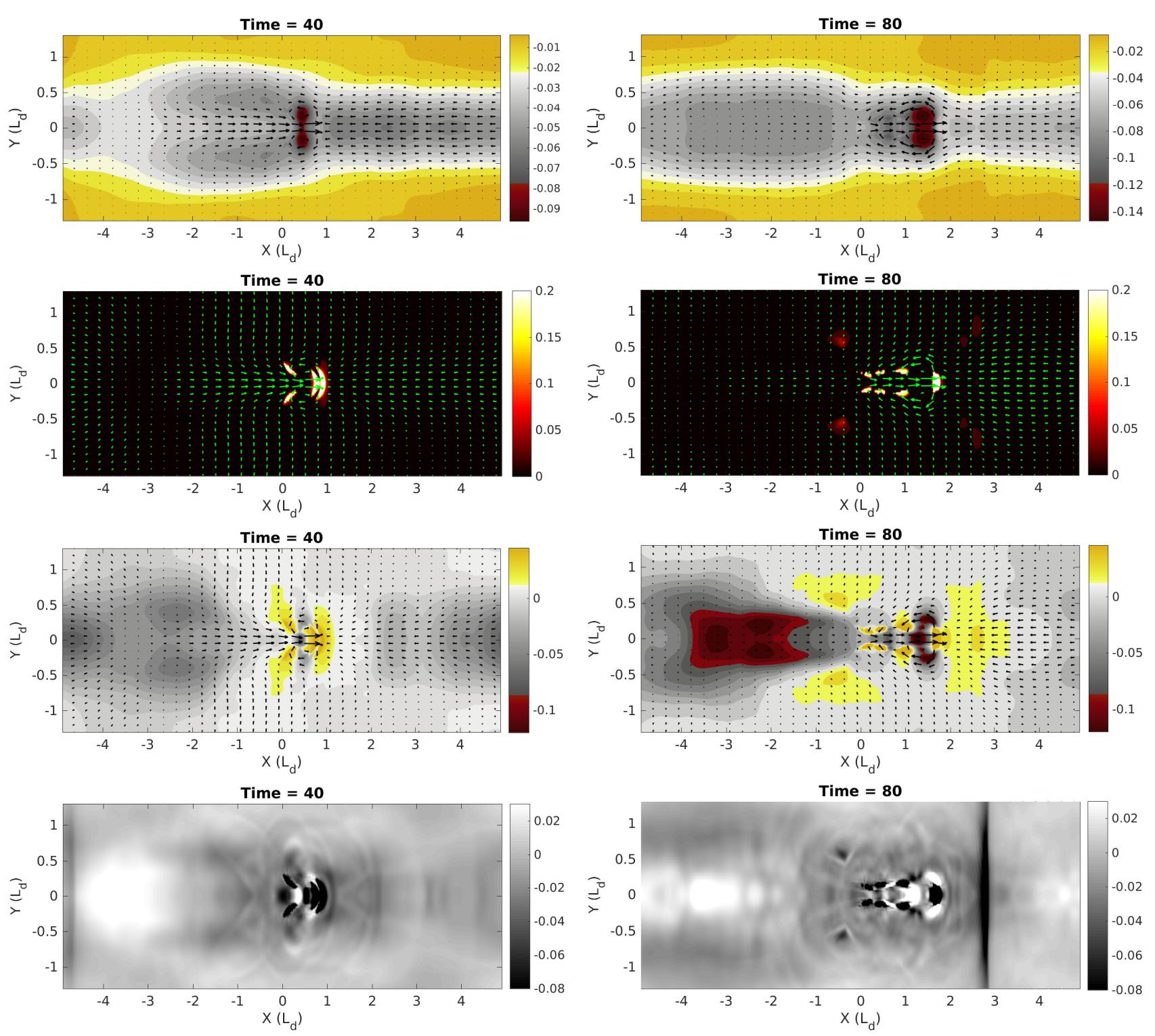

Figure 4. Upper row: Eastward-propagating intense baroclinic dipole as seen in the baroclinic pressure anomaly (colors) and baroclinic velocity (arrows) at times $t=40,80\left(L_{d} / \sqrt{g H_{s}}\right)$, Second row: Corresponding condensation pattern, Third row: Distribution of moisture anomaly (colors) in the lower layer, and corresponding velocity field (arrows), Lower row: Divergence in the lower layer. Initial conditions and parameter values are the same as that of in Fig. 1.

a zonally asymmetric quadrupole vorticity structure arises in the lower layer and its opposite-sign weaker counterpart forms in the upper layer, with corresponding convergence and divergence zones in the lower and upper layers, respectively. The stronger dipole in the western sector of this circulation pattern originates from the Rossby-wave response, and the weaker one in the eastern sector - from the vorticity pattern of the Kelvin wave. The quadrupolar structure also persists for a long time, becoming more zonally dissymmetric at later stages. Westerly and easterly zonal winds, respectively, to the West and to the East of the center of the resulting system feed up moisture and maintain convergence at the center in the lower layer, which is necessary for self-sustained moist convection. The resulting hybrid baroclinic Rossby-Kelvin mode has the same structure as in the classical Gill mechanism of the response of the tropical atmosphere to localized heating Gill (1980), with a notable difference that it is self-sustained and self-propelled slowly eastward. It nevertheless loses its coherence after $\approx 4$ days, shedding then a baroclinic Kelvin wave, and becoming a coherent baroclinic dipole, which continues slow eastward motion without losing coherence for $\approx 11$ days more, convection associated with the dipole covering a distance of about $2 L_{d} \approx 64^{\circ}$. The mean zonal speed of this structure is $\approx 5.5 \mathrm{~ms}^{-1}$, and it undergoes interactions with circumnavigating Kelvin wave packets, which itself engendered. 

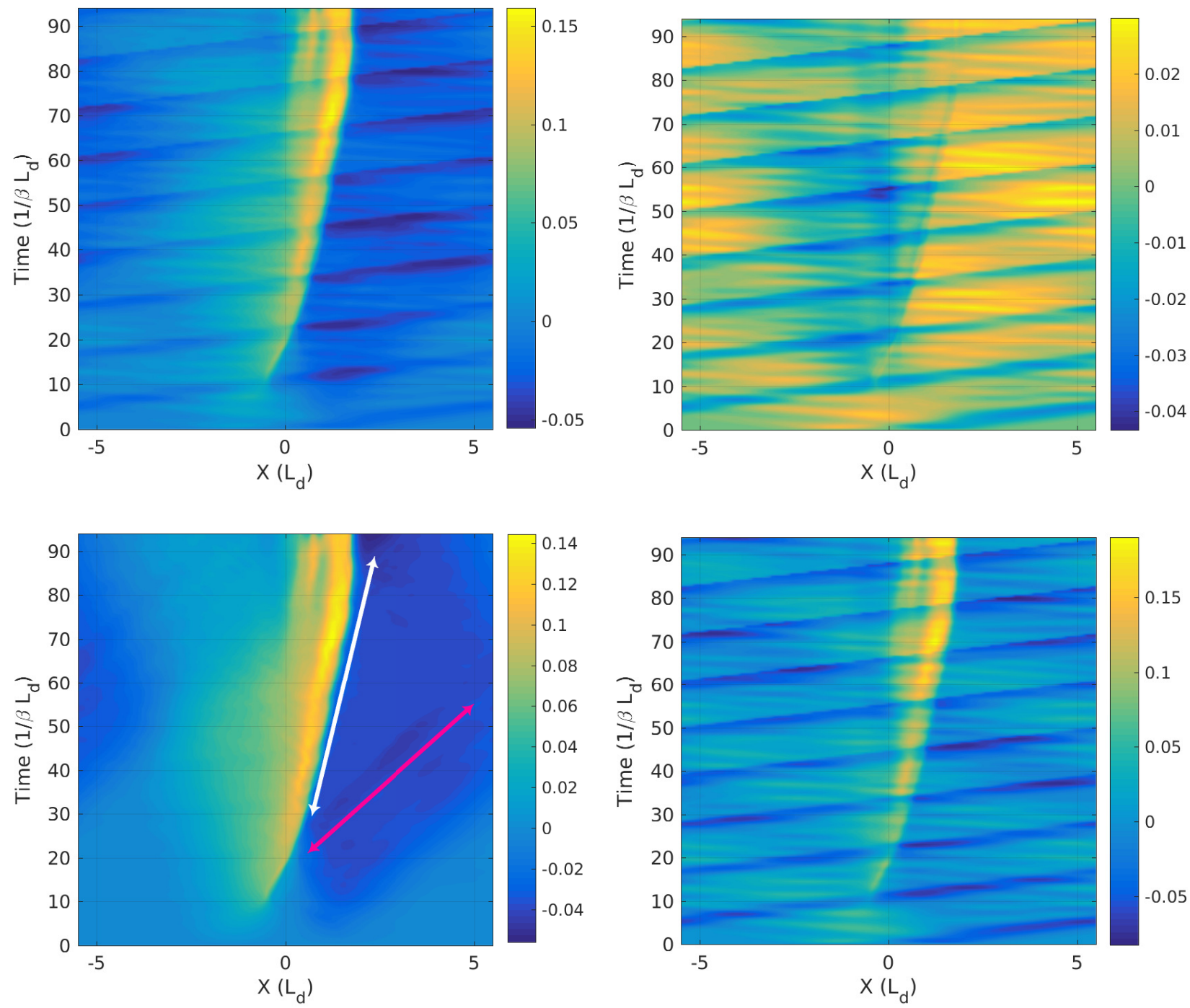

Figure 5. Upper row: Hovmöller diagram of the zonal velocity at the Equator $(y=0)$ in the lower (left panel) and upper (right panel) layers. Lower row: Hovmöller diagram of the baroclinic (left panel) and barotropic (right panel) zonal velocity. White and red arrows indicate the eastward propagation of the dipole and baroclinic Kelvin wave, respectively. Initial conditions are the same as Fig. 1
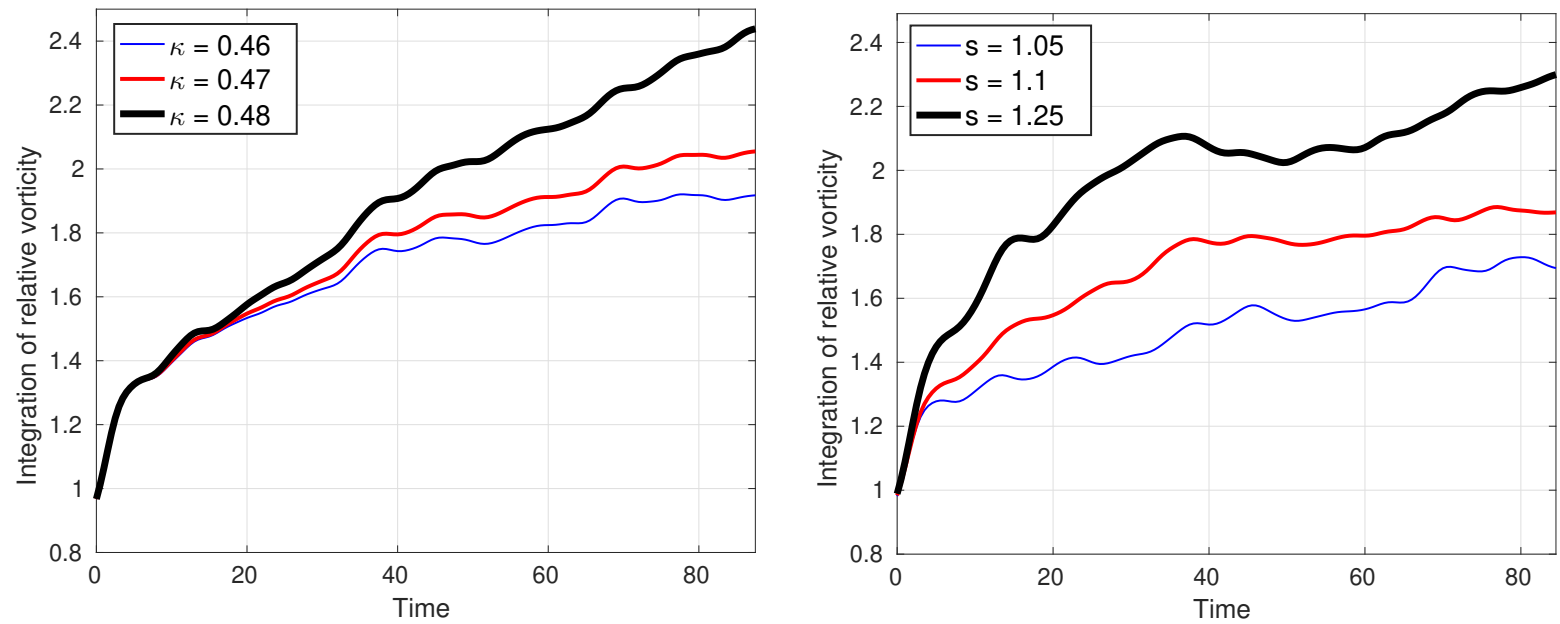

Figure 6. Sensitivity of the overall vorticity produced during equatorial adjustment of a negative pressure anomaly in the lower layer to the intensity of convective activity $\kappa$, which was varied by changing $Q^{s}$, at $s=1.1$, and to stratification $s=\theta_{2} / \theta_{1}$ at $Q_{s}=0.87, \gamma=0.35, H_{2}=0.65$ as seen in the the relative vorticity anomaly integrated over the computational domain. Curves smoothened over the $5 \Delta t$ window. $\kappa=0.47$ and $s=1.1$ correspond to the simulation of the previous figures.

\subsection{Similarities of the dynamical structure emerging in the adjustment process with MJO events}


observational studies allowed to well document various aspects of this inter-seasonal oscillation. We list below some key observational facts on the MJO events, and their counterparts in our experiments:

\section{- Quadrupolar structure with opposite-sign vorticity anomaly in the upper layer}

One of the key manifestations of the MJO events is a robust signature of vorticity quadrupole at both upper and lower layers with opposite signs (Rui and Wang 1990; Kiladis et al. 2005). Wang et al. (2019) clearly identified an opposite-sign vorticity dipole in the upper layers, over the main dipole in the lower layers. Our simulations revealed precisely a structure of this type at the initial stages of adjustment, cf. Figure 1. At later stages it persists as well, but becomes more and more zonally dissymmetric, with weakening front-edge dipole.

- Enhanced dipolar structure in the lower layer, Kelvin wave detachment, and zonal spreading

The anticyclonic component of the lower-layer quadrupole remains prominent in our simulations only at the early stages, because of eventual detachment of the corresponding Kelvin wave. A combination of a forced component of a hybrid Rossby-Kelvin type and a radiative, Kelvin-wave response, was reported in MJO events by Hendon and Salby (1994), and similarly by Dunkerton and Crum (1995). We observe the same scenario at the later stages of adjustment, cf. the left column of Fig. 4. A similar scenario in evolution of potential vorticity of the MJO events, and its corresponding tendencies, were found in observations by Zhang and Ling (2012). The rear dipolar structure dominating the lower-level quadrupole is well documented, cf. (Lau and Phillips 1986; Zhang and Ling 2012; Wang et al. 2019).

The detachment of Kelvin waves from the main MJO events in observations was analyzed by Sobel and Kim (2012). The Hovmöller diagram of the lower-left panel of Fig. 5 resembles much those of Sobel and Kim (2012).

We observe in our simulations a slow zonal westward spreading of the resulting in the adjustment structure which, itself, is moving eastward. The Rossby-wave tail has a tendency to move westward, but the simultaneously developing moist-convection intensifies it, and makes it eventually drift eastward. The center of convection is in the convergent zone, which is in the center of the quadrupole, which is consistent with the MJO structure (Zhang 2005). So the center of the initial quadrupole, which is situated at the eastern part of the Rossby dipole, pulls eastward and the western tail pulls westward, which leads to a zonal spread of the vorticity field. Such deformation resembles the observed evolution of the MJO (Kiladis et al. 2005). At later stages we also observe a baroclinic Rossby wave-like tail extending behind the main dipole, which then interacts with circumnavigating Kelvin wave, cf. Fig. 4, upper panel.

\section{- Westerly and easterly inflows, moisture distribution, and precipitation}

In the lower troposphere (850-hPa level), the episodic anomalously strong westerly surface winds to the West of the large-scale convective center, and anomalous easterly winds to the East of it that last up to a month, are a distinct feature of the MJO (Madden 1986; Sperber 2003; Zhang 2005). This characteristic is reproduced in our simulations, both in the hybrid mode, cf. Fig. 1, and after the Kelvin wave shedding, cf. Fig. 4, third panel, with convergent and divergent zones in the lower layer corresponding to ascending and descending motions, respectively. A regions of enhanced humidity to the East, and depleted humidity to the west of the MJO center are typical, e.g. (Sperber 2003; Kiladis et al. 2005; Adamec and Wallace 2015). We do observe such distribution, cf. Fig. 4, third panel. As regards the precipitation, its typical for MJO events swallowtail structure recovered from observations (Zhang and Ling 2012; Adamec and Wallace 
2015) corresponds, rather, to the overall depression pattern in our simulations, cf. Fig. 2, lower rightmost panel, than to the condensation (which is synonymous to precipitation in the simplest version of the model we are using) of Fig. 4, second row. The reason is that, by construction, our model captures only the deep convection, and related precipitations.

\section{- Propagation speed vs Kelvin-waves phase velocity}

One of the most highlighted features of the MJO, which distinguishes it from equatorial waves, is its slow eastward propagation at average speed of $\approx 5 \mathrm{~ms}^{-1}$ (Weickmann et al. 1985; Knutson et al. 1986; Wheeler and Kiladis 1999). In our simulations, the zonal phase speed of the baroclinic modon-like dipole is $5.6 \mathrm{~ms}^{-1}$, as follows from Fig. 5 that is close to aforementioned observations. The phase-speed of radiative Kelvin-wave response in (Hendon and Salby 1994) was estimated at $\approx 10 \mathrm{~ms}^{-1}$, which is close to the value recovered from the corresponding slope in Fig. 5, lower-right panel, although higher propagating speeds of $15-17 \mathrm{~ms}^{-1}$ for baroclinic convectively coupled Kelvin waves were deduced from observations (Wheeler and Kiladis 1999; Straub and Kiladis 2002). In general, phase speeds of associated Kelvin waves during the active and the suppressed phases of the MJO vary and have a rather broad range, from 10 to $17 \mathrm{~ms}^{-1}$ (Roundy 2008). According to Roundy (2008), the phase speed during the active phases of MJO, over the tropical Indian Ocean, is smaller than that of the suppressed phase over Pacific, and Kelvin waves propagate through the MJO patterns. We also observe Kelvin waves catching up and interacting with the main MJO dipole, cf. Fig. 4, and a difference in phase speeds of the baroclinic Kelvin waves in our simulations, corresponding to attached and detached Kelvin-wave patterns, cf. the change of slope of the MJO track, and its bifurcation at $t \approx 20$ in the lower-right panel of Fig. 5. Observational studies evidence fast eastward propagation of MJO-related signals over the Pacific Ocean, once the MJO convection ceases in the eastern Pacific (Madden and Julian 1972; Hendon and Salby 1994; Milliff and Madden 1996; Weickmann et al. 1997; Matthews 2000; Sperber 2003). The interactions of circumnavigating Kelvin waves with the main MJO pattern, and correlation of these interactions with initiation and dissipation of MJO are reported both in observations and in simulations with the Lagrangian general circulation model (Haertel et al. 2014), a rare "big" model which systematically captures the MJO events (Haertel et al. 2013).

\subsection{Discussion and conclusions}

Relations to and tentative explanations in terms of linear and nonlinear equatorial waves of the MJO have a long record, e.g (Lau and Peng 1987; Chang and Lim 1988; Hendon 1988; Wang and Rui 1990; Salby and Hendon 1994; Maloney and Hartmann 1998; Roundy 2008; Yang and Ingersoll 2011; Wedi and Smolarkiewicz 2010; Yano and Tribbia 2017). (There are also claims that MJO has little to do with waves (Zhang and Ling 2012)). Equatorial adjustment is a generator of waves par excellence. Our experiments on adjustment of lower-troposphere pressure anomalies in the simplest, albeit self-consistent, baroclinic model show a genesis of structures combining Rossby- and Kelvinwave responses and reproducing many of the observed features of the MJO events crudely, but surprisingly well. In a way, the present study allows to reconcile a number of previously existing approaches. The response to the localized depression we observe is a combination of the modon-like structure à la Yano and Tribbia (2017) attached to a Kelvin wave, and forming a hybrid Rossby-Kelvin structure à la Gill (1980). It encompasses a convective activity à la (Majda and Stechmann 2009) in a natural way, through the moisture variable $Q$, and thus allows to track "moisture modes" $a ̀$ la (Sobel and Maloney 2013). The pronounced similarities of the dynamical structures arising during the baroclinic equatorial adjustment with the MJO events give a strong indication that the two processes are strongly related. Of course, the mc2RSW model is over-simplified, which is, at the same time, a weakness and a strength. By construction, it cannot represent the details of the vertical structure, but it captures robustly the main dynamical effects of the moist convection. We should recall that the "big" models struggle to reproduce the MJO, e.g. (Kim et al. 2011), and their results are highly sensitive to the details of 
parameterizations of the moist convection. The robust features of equatorial adjustment found in the present paper could give hints of what to look at and what to look for in more comprehensive models.

We should comment the fact that, compared to the observed life-time of the MJO events ( $\geq 30$ days), the life-time of the MJO-like structure we found is much shorter $(\approx 12$ days $)$, although it is of the same order of magnitude. A possible reason of this discrepancy lies in interactions of the structure with circumnavigating Kelvin and Rossby waves. Indeed, according to the estimates of the phase velocities of linear waves, the barorotropic Kelvin waves make several encounters with the MJO-like structure until it stalls, as distinctly seen in the lower-right panel of Fig. 5. A slower westward-propagating weak barotropic Rossby waves can be also distinguished, and do have encounters with the main structure. The moist barotropic waves are too fast in the mc2RSW model, cf. (Lambaerts et al. 2011b), and their interactions with the main structure in our experiments are, probably, exaggerated. The barotropic waves cannot be easily filtered, as the main structure has a barotropic component, too. We also did not include spatial variations of $Q^{s}$, which can influence the dynamics (Sukhatme 2014), and definitely play a role in the life-cycle of MJO (Kim et al. 2017).

We could not address the important questions of repetitive character of MJO, and of their teleconnections with higher latitudes, e.g. (Wang et al. 2019) in the present equatorial beta-plane adjustment set-up, but the first approach to these problems could be also made in the framework of mc2RSW, considered on the whole sphere. Let us recall in this context that introduction of orographic effects is straightforward and computationally friendly in shallow-water models. Topography will modify around Equator the propagation of waves emitted in the adjustment process, and hence will influence their interactions with the MJO-like structure. As was already said, an improved version of the model, including liquid water (clouds), inter-layer entrainment, vaporization, and precipitation can be used, in order to increase its realism. Let us mention in this connection another element, which was deliberately omitted in the present implementation, but can be important for longer-time simulations and could influence the predictions for the later stages of the adjustment process: the radiative relaxation, i.e. the tendency to radiative-convective equilibrium, which plays a role in the MJO dynamics (Kim et al. 2015). It could be easily included in the model as a simple relaxation, with a typical relaxation time about one month, either to the non-perturbed thicknesses of the layers, in the simplest version, or to an equilibrium profile defined by the liquid water content in the lower layer, in the improved version of (Rostami and Zeitlin 2018).

A. Calculation of the coefficients $A_{ \pm}, B_{ \pm}$

Comparing (2.10) and (2.12) we get:

$$
B_{ \pm}=\frac{d\left(1+s A_{ \pm}\right)}{1+A_{ \pm}}, \quad A_{ \pm}=\frac{B_{ \pm}\left(1+\frac{\kappa}{d}\right)}{1-\kappa}
$$

Notice that in the absence of the moist convection $B_{ \pm} \equiv A_{ \pm}$, and that $1-\kappa$ is non-dimensional moist enthalpy, which should remain positive. Solving this pair of algebraic equations gives:

$$
A_{ \pm}=\frac{(\sigma s-1)}{2} \pm \sqrt{\frac{(\sigma s-1)^{2}}{4}+\sigma},
$$

where we used the notation $\sigma=(d+\kappa) /(1-\kappa)$. In the absence of moist convection $\sigma \equiv d$ and, thus, mist convection leads to effective increase of the ratio $d$, which is not surprising, as it effectively diminishes $H_{1} . B_{ \pm}$is recovered from the first equation in (A.17). It is easy to see that for $s-1=$ $\Delta \ll 1$, which is our case here, $A_{+}=d+\mathcal{O}(\Delta), A_{-}=-1+\mathcal{O}(\Delta)$, in the "dry" configuration and the baroclinic component is approximately the difference between the upper and lower layers. The expression for the square of the phase velocity (2.14) is:

$$
c_{ \pm}^{2}=\left(\frac{(\sigma s+1)}{2} \pm \sqrt{\frac{(\sigma s-1)^{2}}{4}+\sigma}\right)(1-\kappa) .
$$


Acknowledgements This work was supported by the French National Program LEFE-IDAO, and the German Research Foundation within the framework of the CRC 806 (project ID 57444011). Declaration of Interests The authors report no conflict of interest.

\section{References}

Adamec, A., Wallace, J., 2015. Three - dimensional structure and evolution of the moisture field in the mjo. J. Atmos. Sci. 72, $3733-3754$.

Betts, A., Miller, M., 1986. A new convective adjustement scheme. part ii: Single columns tests using gate wave, bomex, atex and arctic air-mass data sets. Q. J. Roy. Met. Soc. 112, 693-762.

Bouchut, F., 2007. Chapter 4: Efficient numerical finite volume schemes for shallow water models. In: Zeitlin, V. (Ed.), Nonlinear Dynamics of Rotating Shallow Water: Methods and Advances. Vol. 2 of Edited Series on Advances in Nonlinear Science and Complexity. Elsevier, pp. 189 - 256.

Bouchut, F., Lambaerts, J., Lapeyre, G., Zeitlin, V., 2009. Fronts and nonlinear waves in a simplified shallow-water model of the atmosphere with moisture and convection. Phys. Fluids 21 (11), 116604.

Bouchut, F., Zeitlin, V., 2010. A robust well-balanced scheme for multi-layer shallow water equations. Disc. Cont. Dyn. Syst. B13, 739-758.

Chang, C.-P., Lim, H., 1988. Kelvin Wave-CISK: A possible mechanism for the 30-50 day oscillations. J. Atmos. Sci. 45 (11), $1709-1720$.

Dunkerton, T. J., Crum, F. X., 1995. Eastward propagating 10 - to 15 - day equatorial convection and its relation to the tropical intraseasonal oscillation. J. Geophys. Res. 100 (D12), 25781-25790.

Fuchs, Z., Raymond, D. J., 2017. A simple model of intraseasonal oscillations. J. Adv. Mod. Earth Sys. 9 (2), $1195-1211$.

Gill, A., 1980. Some simple solutions for heat induced tropical circulation. Q. J. Roy. Met. Soc. 106, 447-462.

Gill, A., 1982. Studies of moisture effects in simple atmospheric models: The stable case. Geophys. Astrophys. Fluid Dyn. 19 , 119.

Haertel, P., Straub, K., Budsock, A., 2014. Transforming circumnavigating Kelvin waves that initiate and dissipate the Madden - Julian Oscillation. Q. J. Roy. Met. Soc. 141, 1586-1602.

Haertel, P., Straub, K., Fedorov, A., 2013. Lagrangian overturning and the Madden - Julian Oscillation. Q. J. Roy. Met. Soc. 140, $1344-1361$.

Heckley, W. A., Gill, A., 1984. Some simple analytical solutions to the problem of forced equatorial long waves. Q. J. Roy. Met. Soc. $110,203-217$.

Hendon, H. H., 1988. A simple model of the 40-50 day oscillation. J. Atmos. Sci. 45 (4), 569-584.

Hendon, H. H., Salby, M. L., 1994. The life cycle of the Madden-Julian Oscillation. J. Atmos. Sci. 51 (15), $2225-2237$.

Hoskins, B. J., Jin, F. F., 1991. The initial value problem for tropical perturbations to a baroclinic atmosphere. Q. J. Roy. Met. Soc. 117, $299-317$.

Katsaros, K., 2001. Evaporation and humidity. In: Steele, J. H. (Ed.), Encyclopedia of Ocean Sciences. Academic Press, Oxford, pp. 870 - 877.

Kiladis, G. N., Straub, K. H., Haertel, P. T., 2005. Zonal and vertical structure of the Madden-Julian Oscillation. J. Atmos. Sci. 62 (8), $2790-2809$.

Kim, D., Ahn, M., Kang, I., DelGenio, A., 2015. Role of longwave cloud - radiation feedback in the simulation of the Madden-Julian Oscillation. J. Climate. 28, 6979-6994.

Kim, D., Kim, H., Lee, M., 2017. Why does the MJO detour the Maritime Continent during austral summer? Geoph. Res. Lett. 44, $2579-2587$.

Kim, D., Sobel, A., Maloney, E., D.M.W., F., Kang, I., 2011. A systematic relationship between intraseasonal variability and mean state bias in agcm simulations. J. Climate. 24, 5506-5520.

Knutson, T. R., Weickmann, K. M., Kutzbach, J. E., 1986. Global-Scale intraseasonal oscillations of Outgoing Longwave Radiation and 250 mb zonal wind during northern hemisphere summer. Mon. Weather Rev. 114 (3), 605-623.

Lambaerts, J., Lapeyre, G., Zeitlin, V., 2011a. Moist versus dry barotropic instability in a shallow-water model of the atmosphere with moist convection. J. Atmos. Sci. 68, 1234-1252.

Lambaerts, J., Lapeyre, G., Zeitlin, V., 2012. Moist versus dry baroclinic instability in a simplified two-layer atmospheric model with condensation and latent heat release. J. Atmos. Sci. 69, 1405-1426.

Lambaerts, J., Lapeyre, G., Zeitlin, V., Bouchut, F., 2011b. Simplified two-layer models of precipitating atmosphere and their properties. Phys. Fluids 23 (4), 046603.

Lau, K.-M., Peng, L., 1987. Origin of low-frequency (intraseasonal) oscillations in the tropical atmosphere. part I: Basic theory. J. Atmos. Sci. 44 (6), 950-972.

Lau, K. M., Phillips, T. J., 1986. Coherent fluctuations of fxtratropical geopotential height and tropical convection in intraseasonal time scales. J. Atmos. Sci. 43 (11), 1164-1181.

Le Sommer, J., Reznik, G. M., Zeitlin, V., 2004. Nonlinear geostrophic adjustment of long-wave disturbances in the shallow-water model on the equatorial beta-plane. J. Fluid Mech. 515, 135-170.

Madden, R. A., 1986. Seasonal variations of the 40-50 day oscillation in the tropics. J. Atmos. Sci. 43 (24), 3138-3158.

Madden, R. A., Julian, P. R., 1972. Description of global-scale circulation cells in the tropics with a 40-50 day period. J. Atmos. Sci. 29 (6), 1109-1123.

Majda, A. J., Stechmann, S. N., 2009. The skeleton of tropical intraseasonal oscillations. Proc. Natl. Acad. Sci. USA 106 (21), $8417-8422$.

Maloney, E. D., Hartmann, D. L., 1998. Frictional moisture convergence in a composite life cycle of the Madden-Julian Oscillation. J. Climate 11 (9), 23872403.

Matsuno, T., 1966. Quasi-geostrophic motions in the equatorial area. J. Met. Soc. Japan 44, $25-43$.

Matthews, A. J., 2000. Propagation mechanisms for the Madden-Julian Oscillation. Q. J. R. Met. Soc. 126 (569), $2637-2651$.

Milliff, R. F., Madden, R. A., 1996. The existence and vertical structure of fast, eastward-moving disturbances in the equatorial troposphere. J. Atmos. Sci. 53 (4), 586-597.

Rostami, M., Zeitlin, V., 2017. Influence of condensation and latent heat release upon barotropic and baroclinic instabilities of atmospheric vortices in a rotating shallow water model on the f-plane. Geoph. Astrophys. Fluid Dyn. 111, 1-31.

Rostami, M., Zeitlin, V., 2018. Improved moist-convective rotating shallow water model and its application to instabilities of hurricane-like vortices. Q. J. Roy. Met. Soc. 144, 1450-1462.

Rostami, M., Zeitlin, V., 2019a. Eastward-moving convection-enhanced modons in shallow water in the equatorial tangent plane. Phys. Fluids 31 , 021701.

Rostami, M., Zeitlin, V., 2019b. Geostrophic adjustment on the equatorial beta-plane revisited. Phys. Fluids 31 (8), 081702.

Rostami, M., Zeitlin, V., 2020. Eastward-moving equatorial modons in moist-convective shallow-water models. Geoph. Astrophys. Fluid Dyn., https://doi.org/10.1080/03091929.2020.1805448.

Roundy, P. E., 2008. Analysis of convectively coupled Kelvin waves in the Indian ocean MJO. J. Atmos. Sci. 65 (4), $1342-1359$.

Rui, H., Wang, B., 1990. Development characteristics and dynamic structure of tropical intraseasonal convection anomalies. J. Atmos. Sci. 47 (3), 357-379.

Salby, M. L., Hendon, H. H., 1994. Intraseasonal behavior of clouds, temperature, and motion in the tropics. J. Atmos. Sci. 51 (15), $2207-2224$.

Schecter, D. A., Dunkerton, T. J., 2009. Hurricane formation in diabatic Ekman turbulence. Q. J. Roy. Met. Soc. 135 (641), 823-838.

Sobel, A., Kim, D., 2012. The MJO - Kkelvin wave transition. Geoph. Res. Lett. 39, L20808.

Sobel, A., Maloney, E., 2013. Moisture modes and the eastward propagation of the MJO. J. Atmos. Sci. 70, $187-192$.

Solodoch, A., Boos, W., Kuang, Z., Tziperman, E., 2011. Excitation of intraseasonal variability in the equatorial atmosphere by Yanai wave groups via WISHEinduced convection. J. Atmos. Sci. 68, 210-225.

Sperber, K., 2003. Propagation and the vertical structure of the Madden - Julian Oscillation. Mon. Weather Rev. 131, $3018-3037$. 
Straub, K. H., Kiladis, G. N., 2002. Observations of a convectively coupled Kelvin wave in the Eastern Pacific ITCZ. J. Atmos. Sci. 59 (1), 30-53. Sukhatme, J., 2014. Low-frequency modes in an equatorial shallow - water model with moisture gradients. Q. J. Roy. Met. Soc. 140, $1838-1846$.

Vallis, G. K., Penn, J., 2019. Convective organization and eastward propagating equatorial disturbances in a simple excitable system.

Wang, B., Rui, H., 1990. Dynamics of the coupled moist Kelvin-Rossby wave on an equatorial $\beta$-plane. J. Atmos. Sci. 47 (4), $397-413$.

Wang, D., Yano, J.-I., Lin, Y., 2019. Madden-Julian Oscillations seen in the Upper-Troposphere vorticity field: Interactions with Rossby wave trains. J. Atmos. Sci. 76 (6), 1785-1807.

Wedi, N. P., Smolarkiewicz, P. K., 2010. A nonlinear perspective on the dynamics of the MJO: Idealized Large-Eddy simulations. J. Atmos. Sci. 67 (4), 1202-1217.

Weickmann, K. M., Kiladis, G. N., Sardeshmukh, P. D., 1997. The dynamics of intraseasonal atmospheric angular momentum oscillations. J. Atmos. Sci. 54 (11), 1445-1461.

Weickmann, K. M., Lussky, G. R., Kutzbach, J. E., 1985. Intraseasonal (30-60 day) fluctuations of Outgoing Longwave Radiation and 250 mb streamfunction during northern winter. Mon. Weather Rev. 113 (6), 941-961.

Wheeler, M., Kiladis, G. N., 1999. Convectively coupled equatorial waves: Analysis of clouds and temperature in the wavenumber-frequency domain. J. Atmos. Sci. 56 (3), 374-399.

Yang, D., Ingersoll, A. P., 2011. Testing the hypothesis that the MJO is a mixed Rossby-gravity wave packet. J. Atmos. Sci. 68, 226-239.

Yano, J.-I., Tribbia, J., 2017. Tropical atmospheric Madden-Julian Oscillation: a strongly nonlinear free solitary Rossby wave? J. Atmos. Sci. 74, $3473-3489$.

Zeitlin, V., 2018. Geophysical Fluid Dynamics: Understanding (almost) Everything with Rotating Shallow Water Models. Oxford University Press, Oxford.

Zhang, C., 2005. Madden-Julian Oscillation. Rev. Geophys. 43 (2).

Zhang, C., Adames, A., Khouider, Wang, B., Yang, D., 2020. Four theories of the Madden-Julian Oscillation. Rev. Geophys.

Zhang, C., Ling, J., 2012. Potential vorticity of the Madden-Julian Oscillation. J. Atmos. Sci. 69 (1), 65-78. 